\title{
Correction to: Words Speak Louder than Actions: Public Responsiveness to Elite Communication
}

\author{
Jon C. Rogowski ${ }^{1}$ Andrew R. Stone ${ }^{1}$
}

Published online: 29 October 2018

(c) Springer Science+Business Media, LLC, part of Springer Nature 2018

\section{Correction to: Political Behavior https://doi.org/10.1007/s11109-018-9497-x}

The original version of this article unfortunately contained a mistake in Figs. 2 and 3. The shaded regions representing $95 \%$ confidence interval are missing in both the figures. The corrected Figs. 2 and 3 are given below.

The original article has been corrected.

The original article can be found online at https://doi.org/10.1007/s11109-018-9497-x.

Jon C. Rogowski

rogowski@fas.harvard.edu

Andrew R. Stone

arstone@g.harvard.edu

1 Department of Government, Harvard University, 1737 Cambridge Street, Cambridge, MA 02143, USA 


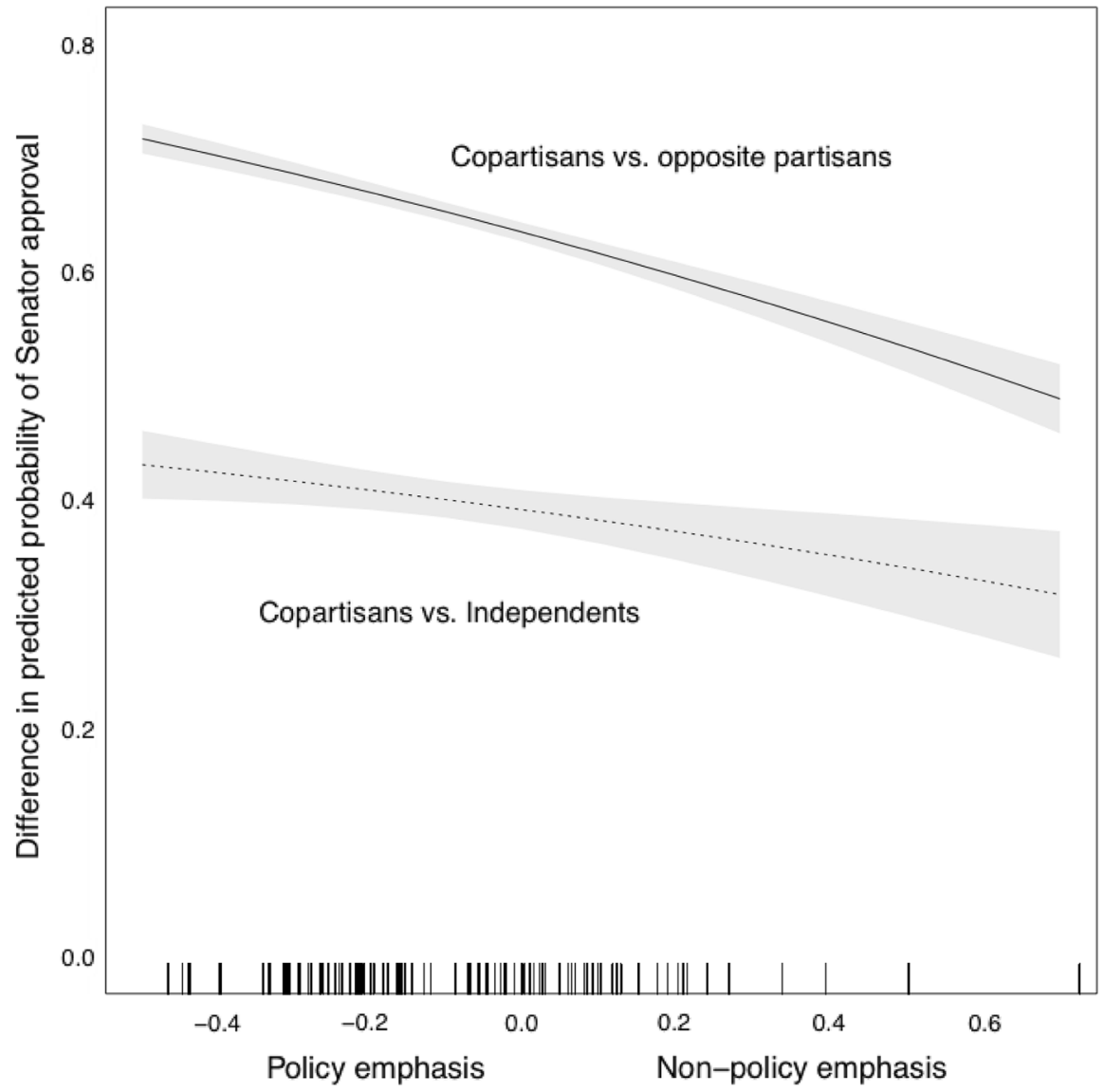

Fig. 2 Effect of communications and partisanship on Senator approval. Plot shows the differences in the predicted probability of approval between a Senator's copartisans and opposite partisans (top line) and a Senator's copartisans and Independents (bottom line). The values along the $x$-axis describe the relative emphasis of Senate communications on policy positions (negative numbers) or non-policy attributes (positive numbers). The rug denotes the distribution of Non-policy emphasis. Large values along the $y$-axis indicate higher levels of predicted approval among copartisans relative to either members of the opposite party or Independents. The shaded regions represent the $95 \%$ confidence intervals 


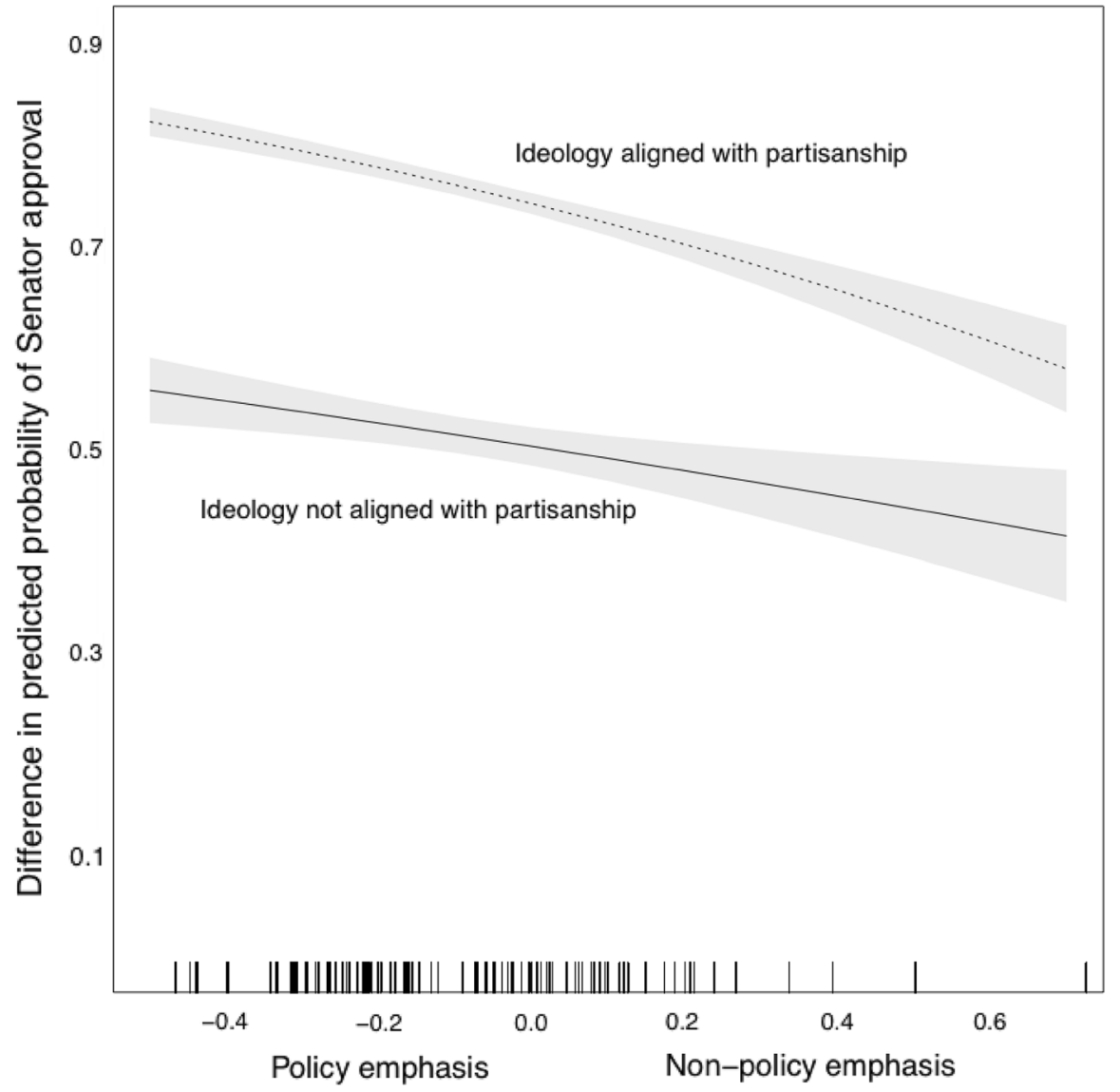

Fig. 3 Partisanship, ideology, and the effect of communications on Senator approval. Plot shows the differences in the predicted probability of approval between a Senator's copartisans and opposite partisans for respondents whose partisan and ideological identities are aligned (top line) and unaligned (bottom line). The values along the $x$-axis describe the relative emphasis of Senate communications on policy positions (negative numbers) or non-policy attributes (positive numbers). The rug denotes the distribution of Non-policy emphasis. Large values along the $y$-axis indicate higher levels of predicted approval among copartisans relative to members of the opposite party. The shaded regions represent the $95 \%$ confidence intervals 